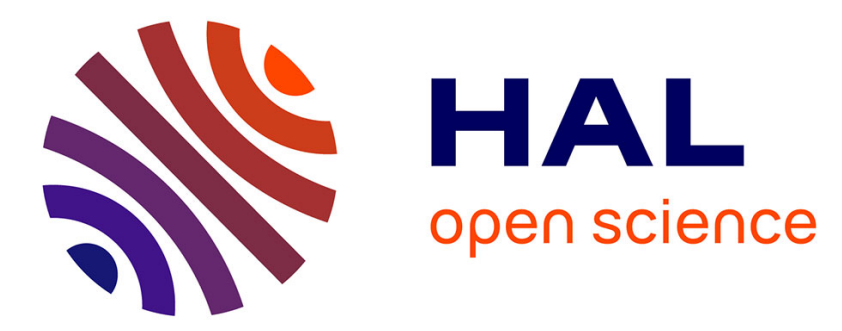

\title{
Mutational equations of the morphological dilation tubes
}

\author{
Luc Doyen, Laurent Najman, Juliette Mattioli
}

\section{To cite this version:}

Luc Doyen, Laurent Najman, Juliette Mattioli. Mutational equations of the morphological dilation tubes. Journal of Mathematical Imaging and Vision, 1995, 5 (3), pp.219 - 230. 10.1007/BF01248373 . hal-00622457

\section{HAL Id: hal-00622457 \\ https://hal.science/hal-00622457}

Submitted on 14 Nov 2014

HAL is a multi-disciplinary open access archive for the deposit and dissemination of scientific research documents, whether they are published or not. The documents may come from teaching and research institutions in France or abroad, or from public or private research centers.
L'archive ouverte pluridisciplinaire HAL, est destinée au dépôt et à la diffusion de documents scientifiques de niveau recherche, publiés ou non, émanant des établissements d'enseignement et de recherche français ou étrangers, des laboratoires publics ou privés. 


\title{
Mutational equations of the morphological dilation
}

\author{
L. Doyen ${ }^{1}$ \& L. Najman ${ }^{1,2} \&$ J. Mattioli ${ }^{2}$ \\ ${ }^{1}$ CEREMADE, Université Paris-Dauphine, \\ Place du Maréchal de Lattre de Tassigny, \\ 75775 Paris Cedex 16, France \\ ${ }^{2}$ L.C.R., Thomson-CSF, \\ Domaine de Corbeville, 91401 Orsay, France
}

\begin{abstract}
The present paper provides some differential results dealing with the morphological dilation of a compact set in the nonregular case. Indeed the evolution of dilated sets with respect to time is characterized through mutational equations which are new mathematical tools extending the concept of differential equations to the metric space of all nonempty compact sets of $\mathbb{R}^{n}$. Using this new tool, we prove that the mutation of the dilation is the normal cone which is a generalization of the classical notion of normal. This result clearly establishes that the dilation transforms this initial set in the direction of the normal at any point of the set. Furthermore, it does not require any regularity assumptions on the compact set.
\end{abstract}

\section{Introduction}

The first class of problems raised in mathematical morphology ([18, Matheron], [28, Schmitt-Mattioli], [29, Serra]) deals with ways of comparing a given shape with a known one, called a structuring element, and to construct and study "morphological operators", such as "dilations", "erosions", "openings", "closings" and morphological filters. Multiscale morphological analysis leads to studying of the evolution of morphological tubes which are set-valued maps $K: t \in \mathbb{R}^{+} \rightsquigarrow K(t) \subset \mathbb{R}^{n}$. In this work, we examine the evolution of the dilation tube: $K(t)=K \oplus t S$ where $K$ (resp. $S$ ) is a subset (resp. a compact convex subset) of $\mathbb{R}^{n}$, and $\oplus$ represents the Minkowski addition [23, Minkowski].

Several studies have investigated the problem of a differential characterization of these operators and especially dilation. But most of these studies 
have been done in a regular context [8, Boomgaard], through partial differential equations ([9, Brocket-Maragos], [1, Alvarez et $A l])$ or through partial differential inclusions $([20,19,21,22$, Mattioli $])$. Let us remark that the slightest dilation of a very regular function (or set) may produce a nonregular result.

Let us recall these previous results in the functional case. The use of the hypograph allows us to consider the functional case in the same way as the set case. We can characterize every function $f: \mathbb{R}^{n} \rightarrow \overline{\mathbb{R}}$ by its hypograph $\mathcal{H} p(f)=\left\{(x, t) \in \mathbb{R}^{n} \times \mathbb{R} \mid f(x) \geq t\right\}$, also called in mathematical morphology subgraph or umbra. In $[1,9]$, the authors have shown that if the following map $(t, x) \mapsto U_{\oplus}(t, x)=(f \oplus t S)(x)$, defined by ${ }^{1}$ $\mathcal{H} p(f \oplus t S)=\mathcal{H} p(f) \oplus t(S \times[-\infty, 0])$, is differentiable, then it is solution of the non-linear parabolic equations:

$$
\left\{\begin{array}{l}
\frac{\partial U_{\oplus}}{\partial t}(t, x)=\sigma\left(S, \frac{\partial U_{\oplus}}{\partial x}(t, x)\right) \\
\text { with } U_{\oplus}(0, x)=f(x)
\end{array}\right.
$$

where $\sigma(S, x)=\sup _{y \in S}\langle x, y\rangle$ is the support function of $S$ and where $<x, y>$ denotes the scalar product on $\mathbb{R}^{n}$.

Here, we wish to establish a more geometrical result on dilation. To this aim, we propose to consider this problem in the mathematical framework of mutational equations ([3, 4, Aubin], [11, 14, Doyen]) which allows one to extend the concept of differential equations to the metric space $\mathcal{K}\left(\mathbb{R}^{n}\right)$ of all non-empty compact subsets of $\mathbb{R}^{n}$. This means that we can characterize the evolution $K(t)$ of a compact geometric set $K$ in a differential manner, without any regularity assumptions on the shape that we deal with, through a kind of differential equation (mutation) of the type

$$
\stackrel{\circ}{K}(t) “=" f(K(t)) \text {. }
$$

where $f$ associates to a domain $K$ a vector field (single-valued or set-valued) and $\stackrel{\circ}{K}(t)$ represents the derivative (in a sense to precise) of $K($.$) at time$ $t$. The essential idea is that the tube $K($.$) evolves in the direction f(K()$.$) ,$ which can depend itself on the tube. Roughly speaking, this means that every point $x \in K(t)$ moves along the trajectory $f(K(t))(x)$. The interest is that this gives a geometrical point of vue of the "trajectory" of the tube $K($.$) , the dynamic of the tube beeing related to the global evolution of K($.$) .$ Furthermore, basic theorems of classical differential systems, as the CauchyLipschitz and Lyapunov ones, can be adapted to mutational equations [15,

\footnotetext{
${ }^{1}$ Under adequate hypothesis we have $(f \oplus t S)(x)=\sup _{y \in S} f(x-t y)$.
} 
12, Doyen]. This provides tools for studying set evolution. Applications to vision based control have emphasized the interest of this approach $[11,13,15$, Doyen].

In the present paper, we show that the morphological tube $K(t)=K \oplus t B$, which corresponds to dilation with respect to the Euclidean unit ball $B$, satisfies the following mutational equation

$$
\left\{\begin{array}{l}
\stackrel{\circ}{K}(t) "=" N_{K(t)} \cap B, \\
K(0)=K
\end{array}\right.
$$

where $N_{K}: x \rightarrow N_{K}(x)$ represents the subnormal cone of the set $K$ at the point $x$ for the Euclidean norm. This result clearly establishes, without any regularity assumptions on the compact set $K$, the intuitive idea that the dilation transforms the initial set $K$ in the direction of the normal at any point of the set. Indeed, when the set $K$ is a regular manifold, the subnormal cone $N_{K}(x)$ is reduced to the half-line spanned by the outward normal $n(x)$ and we rediscover results obtained in the regular case [8]. Figure 2.b. presents some normal cones, and the main result is illustrated on figure 4 .

The paper is organized as follow. First we present dilation by introducing the concept of gauge. Then we give the notion of generalized gradient and we use it to show the relation between gauge and normal cones. We present differential inclusions, which will be used to define mutational derivatives. We give the mutational equation of dilation. Finally, we apply the mutational version of the Cauchy-Lipschitz theorem to compare the evolution of dilations with two different structuring elements.

\section{Dilation, Structuring Element and Gauge}

Specialists of morphological mathematics study shapes or images through a structuring element which allows to compare these shapes. A basic morphological operator is the dilation. There exists several ways to define dilation by a structuring element $S$. Here we propose to introduce this notion using the gauge associated with the structuring element.

The gauge associated with a structuring element $S \subset \mathbb{R}^{n}$ is defined as follows

$$
j_{S}(x):=\inf _{\lambda \geq 0, x \in \lambda S} \lambda .
$$

We can see at once that the following characterization of $S$ holds

$$
x \in S \Longleftrightarrow j_{S}(x) \leq 1 .
$$

If $S$ is the Euclidean unit ball $B$ of $\mathbb{R}^{n}$ centered at the origin, the gauge is identical to the Euclidean norm. More generally, when the set $S$ is compact, convex, with non-empty interior and symmetric with respect to the origin, 
then the gauge defines a norm (see [5, Aubin]). In this case, we can introduce a distance associated with $S$ defined by

$$
d^{S}(x, y):=j_{S}(x-y) .
$$

One can check that these distances are equivalent ${ }^{2}$ to the usual one (Euclidean) which corresponds to the choice of $S=B$, the Euclidean unit ball of $\mathbb{R}^{n}$, centered at the origin.

Through the gauge, we can also define a distance between a set $K$ and a point $x$ by

$$
d_{K}^{S}(x):=\inf _{y \in K} j_{S}(x-y),
$$

and the projection $\Pi_{K}^{S}(x)$ of $x$ on $K$ associated with $S$ as follows

$$
\Pi_{K}^{S}(x):=\left\{z \in K \mid j_{S}(x-z)=d_{K}^{S}(x)\right\} .
$$

We can easily state that

$$
\left\|d_{K}^{S}(x)-d_{K}^{S}(y)\right\| \leq j_{S}(x-y) .
$$

Furthermore, when the set $S$ has a non-empty interior, we can check that the map

$$
x \mapsto d_{K}^{S}(x)
$$

is Lipschitz, i.e. there exists $\alpha \in \mathbb{R}$ such that $\left|d_{K}^{S}(x)-d_{K}^{S}(y)\right| \leq d^{S}(x, y) \leq$ $\alpha d(x, y)$ for all $(x, y) \in \mathbb{R}^{2}$.

The dilation, at scale $t>0$, of a closed set $K \subset \mathbb{R}^{n}$ by a structuring element $S$ is defined as follows (see fig. 1)

$$
K_{S}(t):=\left\{x \in \mathbb{R}^{n} \mid d_{K}^{S}(x) \leq t\right\}
$$

and is equal to

$$
K_{S}(t)=K \oplus t S=\{k+t s \mid k \in K, s \in S\} .
$$

\section{Generalized gradient and subnormal cone}

In the sequel, we assume that $S$ is a compact convex set symmetric with respect to the origin, with non-empty interior. Generally, the distance function $x \mapsto d_{K}^{S}(x)$ is not differentiable in the classical sense (except when $K$ is convex). But, since it is a Lipschitz map, we can consider its generalized gradient.

\footnotetext{
${ }^{2}$ Two distances $d_{1}$ and $d_{2}$ are equivalent if there exists $(\alpha, \beta) \in \mathbb{R}^{2}$ such that $\alpha d_{1}(x, y) \leq$ $d_{2}(x, y) \leq \beta d_{1}(x, y)$ for all $(x, y) \in \mathbb{R}^{n} \times \mathbb{R}^{n}$
} 

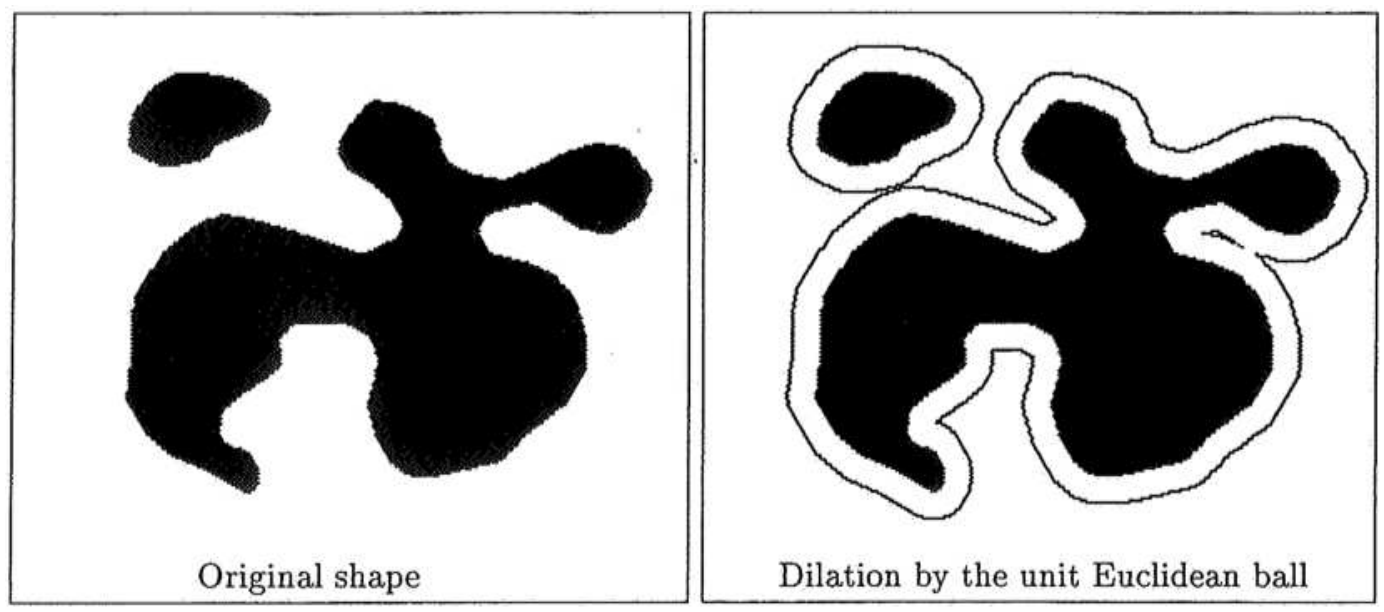

Figure 1: Dilation by the unit Euclidean ball in $\mathbb{R}^{2}$

\subsection{Generalized gradient}

A Lipschitz map $f$ is almost everywhere differentiable. Furthermore we can extend, in a set-valued way, the notion of gradient at any point of the domain of $f$ (see for instance [7, Aubin-Frankowska], [10, Clarke], or [25, Rockafellar-Wets]). We have the following definition:

Definition 3.1 Let $f: \mathbb{R}^{n} \rightarrow \mathbb{R}$ be a Lipschitz map, and $x$ be in $\mathbb{R}^{n}$.

- The circatangent epi-derivative of $f$ at $x$ in the direction $v$ is

$$
C_{\uparrow} f(x)(v):=\limsup _{h \rightarrow 0^{+}, x^{\prime} \rightarrow x} \frac{f\left(x^{\prime}+h v\right)-f\left(x^{\prime}\right)}{h} .
$$

- The generalized gradient of $f$ at $x$ is

$$
\partial f(x):=\left\{p \in \mathbb{R}^{n} \mid C_{\uparrow} f(x)(v) \geq<p, v>, \forall v \in \mathbb{R}^{n}\right\} .
$$

Remark: We can see at once that, if $f$ is $\mathcal{C}^{1}$, then the generalized gradient $\partial f(x)$ is reduced to a singleton, and we have $\partial f(x)=\{\nabla f(x)\}$.

Example: Let $f: \mathbb{R} \rightarrow \mathbb{R}$ be defined by $f(x)=|x|$ for all $x \in \mathbb{R}$. Then the generalized gradient $\partial f$ of $f$ is equal to

$$
\partial f(x)=\left\{\begin{array}{cc}
1 & \text { if } x>0 \\
-1 & \text { if } x<0 \\
{[-1,1]} & \text { if } x=0
\end{array}\right.
$$


As described in [7, Aubin-Frankowska], the set-valued map ${ }^{3}$

$$
x \rightsquigarrow \partial f(x)
$$

satisfies some nice properties that we list below.

Proposition 3.2 Let $f: \mathbb{R}^{n} \rightarrow \mathbb{R}$ be a Lipschitz map and $x$ be in $\mathbb{R}^{n}$. Then

$$
\partial f(x)=\overline{c o}\left(\partial^{*} f(x)\right)
$$

where

$$
\partial^{*} f(x)=\limsup _{x_{n} \rightarrow x}\left\{\nabla f\left(x_{n}\right)\right\},
$$

$\overline{c o}$ represents the closed convex hull, and $\lim \sup _{p} A_{p}$ is the set of all cluster points ${ }^{4}$ of the sequence of sets $A_{p} \subset \mathbb{R}^{n}$.

We can characterize the continuity of a set-valued map in several ways. We recall here the concept of upper-semicontinuity of a set-valued map.

Definition 3.3 (Upper-semicontinuity) Let $X$ be a normed space, and $F$ be a set valued map from $X$ into $X$. The map $F$ is said to be uppersemicontinuous at $x_{0}$ if

$$
\forall \epsilon>0, \exists \eta>0 \text { such that } \forall x \in\left\{x_{0}\right\} \oplus \eta B, F(x) \subset F\left(x_{0}\right) \oplus \epsilon B \text {, }
$$

where $B$ is the unit ball of $X$.

Proposition 3.4 Let $f: \mathbb{R}^{n} \rightarrow \mathbb{R}$ be a Lipschitz map. The set-valued map

$$
x \rightsquigarrow \partial f(x)
$$

is upper semicontinuous with convex compact values.

\subsection{Distance function and sub-normal cone}

In the sequel, we always assume that $K$ is a closed subsed of $\mathbb{R}^{n}$. The distance function $x \mapsto d_{K}^{S}(x)$ is a Lipschitz map. Hence, we can consider its circatangent epi-derivative and its generalized gradient. To compute the generalized gradient of $d_{K}^{S}$, we need two lemmas which are stated and proved in appendix. We then have the following propositions.

\footnotetext{
${ }^{3}$ A set-valued map $F$ from a space $X$ to a space $Y$ is characterized by its graph $\operatorname{Graph}(F)$ defined as follows

$$
\operatorname{Graph}(F)=\{(x, y) \in X \times Y, y \in F(x)\} .
$$

${ }^{4}$ We have $\lim \sup _{p} A_{p}=\cap_{N \geq 1} \overline{\cup_{p \geq N} A_{p}}$
} 
Proposition 3.5 Consider a compact convex symmetric with respect to the origin set $S \subset \mathbb{R}^{n}$ with non-empty interior such that $j_{S}$ is uniformly differentiable. Then, for every $x \in K^{c}$,

$$
\partial d_{K}^{S}(x)=c o\left(\bigcup_{z \in \Pi_{K}^{S}(x)} \nabla j_{S}(x-z)\right) .
$$

The proof of all the theorems and propositions of this paper are given in appendix.

Corollary 3.6 Let $B$ be the Euclidean unit ball of $\mathbb{R}^{n}$. Then, for every $x \in K^{c}$,

$$
\partial d_{K}^{B}(x)=c o\left(\frac{x-\Pi_{K}^{B}(x)}{d_{K}^{B}(x)}\right) .
$$

The the distance function allows us also to define the notions of tangent and normal spaces of a set $K$ at a point $x$ without any regularity assumptions on $K$.

Definition 3.7 (Tangent and normal spaces) Let $K$ be a closed subset of $\mathbb{R}^{n}, S$ a compact convex symmetric (with respect to the origin) set with non-empty interior and $x$ an element of $\mathbb{R}^{n}$.

- The external circatangent cone of $K$ at $x$ associated with $S$ is

$$
C_{K}^{S}(x):=\left\{v \mid C_{\uparrow} d_{K}^{S}(x)(v) \leq 0\right\} .
$$

- The external subnormal cone of $K$ at $x$ associated with $S$ is the negative polar cone of $C_{K}^{S}(x)$ i.e.

$$
N_{K}^{S}(x):=C_{K}^{S}(x)^{-}=\left\{p \mid \forall v \in C_{K}^{S}(x),<p, v>\leq 0\right\} .
$$

Examples of these cones are presented in figure 2 .

Remark: We denote by $C_{K}(x), N_{K}(x)$ the cones associated with the Euclidean norm (i.e. $S=B$ ).

Let us point out here that, when $x$ belongs to $K$ and when the structuring element is the Euclidean unit ball, the external circatangent cone coincides with the usual Clarke cone i.e.

$$
C_{K}(x)=\left\{v \mid \lim _{h \rightarrow 0, x^{\prime} \rightarrow x} \frac{d_{K}^{B}\left(x^{\prime}+h v\right)}{h}=0\right\} .
$$


Let us also make precise that, when the set $K$ is regular ${ }^{5}$, this tangent cone corresponds to the classical definition of the tangent space, and when $K$ is convex, it is the definition used in convex analysis i.e. $C_{K}(x)=\overline{\bigcup_{h>0} \frac{K-x}{h}}$. Let us mention a link between the generalized gradient of the distance functions to $K$ and the subnormal cone. To do this, let us recall respectively the definitions of the polar set $S^{\star}$ of a structuring element $S$.

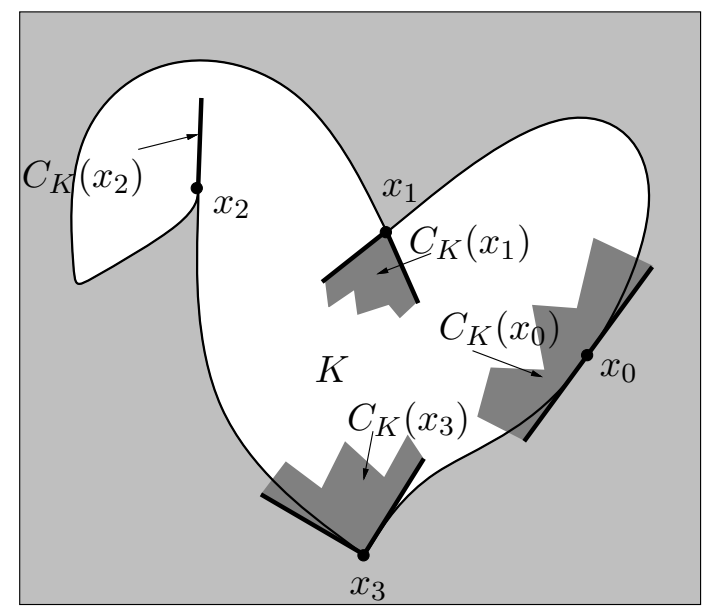

(a) Circatangent Cones at different points of a shape $K$

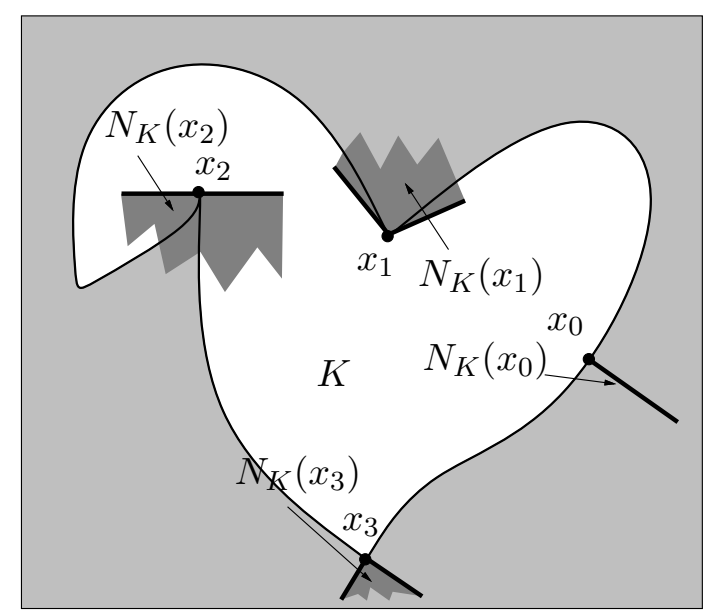

(b) Normal Cones at different points of a shape $K$

Figure 2: Circatangent and Normal cones

Proposition 3.8 Let $K$ be a compact subset of $\mathbb{R}^{n}$ and $S$ a compact, convex and symmetric (with respect to the origin) set with non empty interior. For any $x \in \mathbb{R}^{n}$, we have

$$
\partial d_{K}^{S}(x) \subset N_{K}^{S}(x) \cap S^{\star}
$$

where

$$
S^{\star}:=\left\{p \in \mathbb{R}^{n} \mid \sup _{x \in S}<p, x>\leq 1\right\}
$$

is called the polar set of $S$.

Corollary 3.9 Let $K$ be a compact subset of $\mathbb{R}^{n}$ and $B$ the Euclidean unit ball of $\mathbb{R}^{n}$. For any $x \in \mathbb{R}^{n}$, we have

$$
\partial d_{K}^{B}(x) \subset N_{K}(x) \cap B .
$$

\footnotetext{
${ }^{5}$ i.e. $K=\{x \mid g(x)=0\}$ with $g$ differentiable
} 


\section{Differential Inclusions}

This section is a brief survey of differential inclusion theory. We recall basic facts about it, and focus especially on the reachable maps which will serve as "tangent line" in the definition of mutational derivatives. For more details on the differential inclusion theory, see [6, Aubin-Cellina], [2, Aubin] or [17, Frankowska].

Differential inclusion are natural in control systems. Such systems are often governed by a family differential equation $x^{\prime}(t)=f(x(t), u(t))$ where $u(t) \in$ $U(x(t))$. The single-valued map $f$ describes the dynamics of the system: It associates with state $x$ of the system and the control $u$ the velocity $f(x, u)$ of the system. The set-valued map $U$ describes a feedback map assigning to the state $x$ the subset $U(x)$ of admissible controls. If we put $F(x):=$ $f(x, U(x))=\{f(x, u)\}_{u \in U(x)}$, then the control system is governed by the differential inclusion $x^{\prime}(t) \in F(x(t))$.

Let us describe the (non deterministic) dynamics of a system by a set-valued map $F$ from the state space $\mathbb{R}^{n}$ to itself. We consider initial value problems (or Cauchy problems) associated to differential inclusion

$$
\text { for almost all } t \in[0, T], \quad x^{\prime}(t) \in F(x(t))
$$

satisfying the initial condition $x(0)=x_{0}$.

Definition 4.1 [6] Let $F$ be a set-valued map from $\mathbb{R}^{n}$ to $\mathbb{R}^{n}$. A map $x:[0, T] \rightarrow \mathbb{R}^{n}$ is a solution of the differential inclusion

$$
x^{\prime} \in F(x)
$$

if $x(\cdot)$ is absolutely continuous and

$$
x^{\prime}(t) \in F(x(t)) \text { almost everywhere on }[0, T] .
$$

We denote by $\mathcal{S}\left(x_{0}\right)$ or by $\mathcal{S}_{F}\left(x_{0}\right)$ the (possibly empty) set of solutions to differential inclusion (2).

Definition 4.2 (Solution Map) We shall say that the set-valued map $\mathcal{S}$ defined by $\operatorname{Dom}(F) \ni x \mapsto \mathcal{S}(x)$ is the solution map of $F$ (or of differential inclusion (2).)

We associate with the solution map $\mathcal{S}_{F}: X \rightsquigarrow \mathcal{C}\left(0, \infty ; \mathbb{R}^{n}\right)$ of the differential inclusion (2) the reachable map, (or flow, or set-valued semi-group) defined in the following way:

Definition 4.3 For any $t \geq 0$, we denote by $\vartheta_{F}(t, x):=\left(\mathcal{S}_{F}(x)\right)(t)$ the set of states $x(t)$ reached from $x$ through differential inclusion (2) and by

$$
\vartheta_{F}(t, K):=\left(\mathcal{S}_{F}(K)\right)(t)
$$

the set of states $x(t)$ reached from $K$ by solutions $x(\cdot) \in \mathcal{S}_{F}(x)$. It is called the reachable map. 
The reachable map $\vartheta_{F}(t, x)$ enjoys the semigroup property:

$$
\forall t, s \geq 0, \vartheta_{F}(t+s, x)=\vartheta_{F}\left(t,\left(\vartheta_{F}(s, x)\right)\right.
$$

For the time being, let us mention that these maps are closed.

\section{Mutational equation of the dilation}

\subsection{Mutations of tube}

The mathematical framework that we describe now, is inspired from ideas introduced in shape optimization (shape design) where one tries to optimize a criteria defined with respect to a geometric domain. The need for necessary conditions of optimality implies the definition of a gradient with respect to the domain. But generally, the solution, if it exists, is not regular and we have to face the non vectorial structure of the working space. This leads to the adaptation of the notion of perturbation of a domain. The situation is similar if we search for a derivative with respect to time of an evolving domain.

The mathematical framework of mutational equations [4, Aubin] allows us to extend the concept of differential equations to the metric space $\mathcal{K}(E)$ of all non-empty compact subsets of $\mathbb{R}^{n}$.

For defining mutational equations, we supply the space $\mathcal{K}\left(\mathbb{R}^{n}\right)$ with a distance $d$ (for instance the Hausdorff distance given by $\rho\left(K_{1}, K_{2}\right)=\sup \left\{d_{K_{1}}(x)-\right.$ $\left.\left.d_{K_{2}}(x)\right\}\right)$.

We next associate with any set-valued map $F: \mathbb{R}^{n} \rightsquigarrow \mathbb{R}^{n}$, a transition (a reachable map) $\vartheta_{F}(h, K)$. The "curve" $h \mapsto \vartheta_{F}(h, K)$ plays the role of the half lines $h \mapsto x+h v$ for defining differential quotients. Indeed, the classical definition of a derivative is the following: a map $g: \mathbb{R} \rightarrow \mathbb{R}$ is derivable if there exists a vector $u$ such that

$$
\lim _{h \rightarrow 0^{+}}\left\|\frac{g(x+h)-(g(x)+h u)}{h}\right\|=0
$$

We translate this formula by replacing the half-line $\{g(x)+h u \mid h \geq 0\}$ by the reachable map, and the norm by the distance.

Definition 5.1 (Mutation) Let $F$ be a set-valued map from $\mathbb{R}^{n}$ into $\mathbb{R}^{n}$. Consider a tube $K: \mathbb{R}^{+} \rightsquigarrow \mathbb{R}^{n}$. If, for a given $t \in \mathbb{R}^{+}$, the set-valued map $K(\cdot)$ satisfies

$$
\lim _{h \rightarrow 0^{+}} \frac{d\left(\vartheta_{F}(h, K(t)), K(t+h)\right)}{h}=0,
$$

we shall say that $F$ is a mutation at time $t$ of the tube $K$ and we shall write

$$
\stackrel{\circ}{K}(t) \ni F .
$$




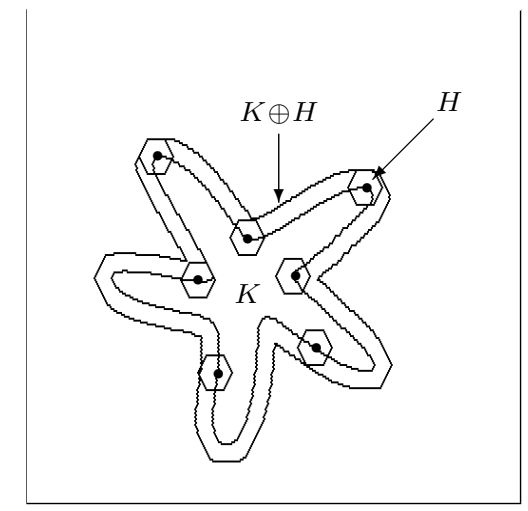

Figure 3: Dilation by an hexagone $H, \stackrel{\circ}{K}(t) \ni H$, where $K(t)=K \oplus t H$.

Example: Let us point out, through a simple example, that the uniqueness of the mutation is not ensured, which justifies the notation. Indeed, consider the constant tube in $\mathbb{R}^{2}$

$$
K(t)=B
$$

where $B$ is the Euclidean unit ball.

It is clear that

$$
0 \in \stackrel{\circ}{K}(t), \forall t .
$$

But we can also check that the Lipschitz map defined by

$$
\varphi(x, y)=(-y, x)
$$

satisfies

$$
\varphi \in \stackrel{\circ}{K}(t)
$$

\subsection{Mutational equations for dilation}

In this section we give three characterizations of the mutation of the dilation tube. These results will give more and more accurate information on how is the instantaneous deformation for dilation.

Let $\mathcal{F}\left(\mathbb{R}^{n}\right)$ the family of set-valued maps from $\mathbb{R}^{n}$ to $\mathbb{R}^{n}$. Now, if we consider a map $f: \mathbb{R}^{+} \times \mathcal{K}(E) \mapsto \mathcal{F}\left(\mathbb{R}^{n}\right)$ associating with a pair $(t, K)$ a set-valued map $x \rightsquigarrow f(t, K)(x)$, we can define a mutational equation for tubes

$$
\stackrel{\circ}{K}(t) \ni f(t, K(t)), \forall t \geq 0
$$

As a first result, dealing with the dilation, we can check the following proposition (see fig. 3): 
Proposition 5.2 Let $K$ be a compact subset of $\mathbb{R}^{n}$ and $S$ a compact, convex, and symmetric set. The dilation tube $K_{S}(t)=K \oplus t S$ associated with the structuring element $S$ satisfies

$$
\left\{\begin{array}{l}
\stackrel{\circ}{K}_{S}(t) \ni S \\
K(0)=K .
\end{array}\right.
$$

But we can be more precise in the following way. Indeed we prove here that the dilation by the Euclidean unit ball satisfies the following mutational equation which describes that the evolution of the dilation can be reduced to the evolution in the normal directions (see fig. 4).

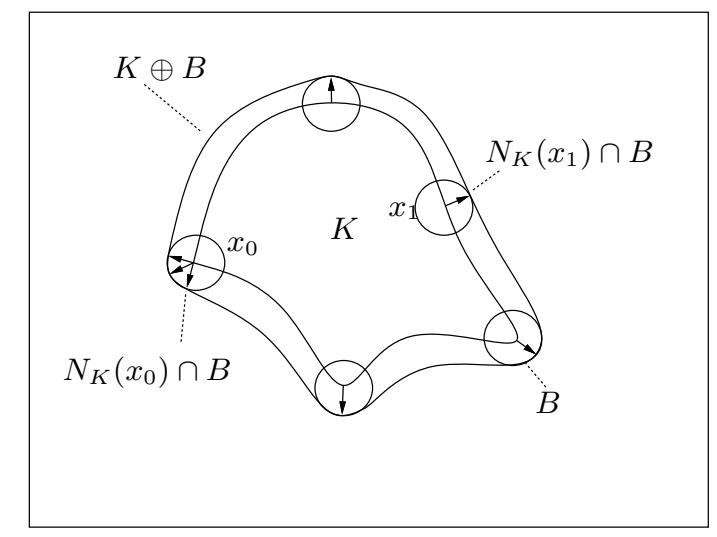

Figure 4: Dilation by an Euclidean ball. We only need the normal cone to obtain the dilated set.

Proposition 5.3 Let $K$ be a compact subset of $\mathbb{R}^{n}$ and $B$ be the Euclidean unit ball of $\mathbb{R}^{n}$. The dilation tube $K(t)=K \oplus t B$ satisfies

$$
\left\{\begin{array}{l}
\stackrel{\circ}{K}(t) \ni \partial d_{K(t)}^{B} \\
K(0)=K .
\end{array}\right.
$$

Using this proposition, we can prove the following theorem:

Theorem 5.4 Let $K$ be a compact subset of $\mathbb{R}^{n}$ and $B$ be the Euclidean unit ball of $\mathbb{R}^{n}$. The dilation tube $K(t)=K \oplus t B$ satisfies

$$
\left\{\begin{array}{l}
\stackrel{\circ}{K}(t) \ni N_{K(t)} \cap B \\
K(0)=K .
\end{array}\right.
$$


This theorem is illustrated by figure 2.b. and figure 4 .

Remark: Using demonstration of proposition 5.3, we can show that

$$
\forall A \text { such that } \partial d_{K}^{B}(.) \subset A \subset B, \stackrel{\circ}{K}(.) \ni A
$$

Recapitulatory: We have obtained three useful mutations of the Euclidean dilation:

$$
\begin{array}{lll}
\stackrel{\circ}{K}(t) & \ni & B \\
\stackrel{\circ}{K}(t) & \ni & N_{K(t)} \cap B \\
\stackrel{\circ}{K}(t) & \ni & \partial d_{K(t)}^{B}
\end{array}
$$

\subsection{Comparison between dilations}

The mutational approach gives a formalism to represent the evolution of tubes. But within this framework, we can also adapt fundamental theorems of the ordinary differential equations as the Nagumo or Cauchy-Lipschitz ones ([4, Aubin], [12, Doyen]), and the Lyapunov method. We use here the Cauchy-Lipschitz version for tubes to estimate the difference between the evolution of dilations governed by distinct structuring elements $S_{1}$ and $S_{2}$. We recall first the extension of the Cauchy-Lipschitz theorem for tubes which allows to compare the evolution of sets with respect to the initial conditions and to dynamics. We shall denote by $\operatorname{LIP}\left(E, \mathbb{R}^{n}\right)$ the family of Lipschitzean set-valued maps from a subset $E$ of $\mathbb{R}^{n}$ into $\mathbb{R}^{n}$.

For $\psi:[0,1] \times \mathbb{R}^{n} \rightarrow \mathbb{R}^{n}$, we note

$$
\|\psi\|_{\Lambda}:=\sup _{h \in[0,1], x \neq y} \frac{d(\psi(h, x), \psi(h, y))}{d l(x, y)}<+\infty
$$

and for a mutable tube $H$ and a mutation $\sigma$, we set

$$
d_{\text {Lip }}(\sigma \stackrel{\circ}{H}(s)):=\inf _{\tau \in \stackrel{\circ}{H(s)}} d l(\sigma, \tau)
$$

Theorem 5.5 (Cauchy-Lipschitz) Let $E$ be a compact subset of $\mathbb{R}^{n}$. Let $H:[0, T] \rightarrow \mathcal{K}(E)$ be a continuous tube having a mutation $\stackrel{\circ}{H}(\cdot) \in \operatorname{LIP}\left(E, \mathbb{R}^{n}\right)$ and $f: \mathcal{K}(E) \rightsquigarrow L I P\left(E, \mathbb{R}^{n}\right)$ be a map such that

$$
\left\{\begin{aligned}
i) & \forall K \in \mathcal{K}(E), \quad f(K) \text { is nonempty closed, } \\
\text { ii) } & f \text { is } \text {-Lipschitz, } \\
\text { iii) } & \|f\|=\sup _{K \in \mathcal{K}(E)} \sup _{\psi \in f(K)}\|\psi\|_{\Lambda}<+\infty \\
\text { iv }) & t \rightarrow \gamma(t)=d_{L I P}(H(t), f(H(t)) \text { is integrable on }[0, T] .
\end{aligned}\right.
$$


Let us set

$$
\eta(t)=e^{(\|f\|+k) t} d\left(K_{0}, H(0)\right)+\int_{0}^{t} e^{(\|f\|+k)(t-s)} d_{\text {Lip }}(\stackrel{\circ}{H}(s), f(H(s))) d s .
$$

Then, from any $K_{0} \in \mathcal{K}(E)$, starts a unique solution $K(\cdot)$ to the mutational inclusion $\stackrel{\circ}{K} \ni f(K)$ and we have

$$
d(K(t), H(t)) \leq \eta(t) .
$$

We can apply this result to compare the evolution of two dilations

$$
K_{S_{1}}(t)=K \oplus t S_{1} \text { and } K_{S_{2}}(t)=K \oplus t S_{2} .
$$

Proposition 5.6 Let $S_{1}, S_{2}$ be two compact, convex, and symmetric sets of $\mathbb{R}^{n}$. We obtain

$$
d l\left(K_{S_{1}}(t), K_{S_{2}}(t)\right) \leq t d\left(S_{1}, S_{2}\right) .
$$

\section{Examples:}

1. Taking in $\mathbb{R}^{2}$, the structuring elements $S_{1}=B$ and $S_{2}=[-1,1] \times$ $[-1,1]$, we obtain

$$
d\left(K_{S_{1}}(t), K_{S_{2}}(t)\right) \leq t(\sqrt{2}-1) .
$$

2. Taking in $\mathbb{R}^{2}$, the structuring elements $S_{1}=B$ and $S_{2}$ the unit hexagonal structuring element, we obtain

$$
d\left(K_{S_{1}}(t), K_{S_{2}}(t)\right) \leq t\left(1-\frac{\sqrt{3}}{2}\right) .
$$

\section{Conclusion and discussion}

In this paper, we gave some different characterizations of the dilation tube of non regular domains in the framework of mutational calculus [4]. We especially focus on the interest of the normal cone (of non regular analysis). It appears that the mutational result involving the normal cone can be generalized to any convex compact symmetric with respect to the origin structuring element S $[24,16]$; in particular, we found

$$
\stackrel{\circ}{K}(t) \ni N_{K(t)}^{S} \cap S
$$

but for the convenience of the reader, we gives here only results and proofs in the case of the Euclidean unit ball.

By computing the mutational derivatives of dilation, we expect to find the set of directions minimal to obtain the dilated set from the original one. 
Our next goal is to translate this continuous result to a grid, in order to produce an efficient algorithm for computing dilations. Our simplest result (formula 3) means that we need all the structuring element for computing the dilated set: this is the simplest algorithm one can think of, and it is widely used in mathematical morphology. But with our finest result (formula 4), we expect to restrict the directions needed, in a similar way to $[26,27$, Schmitt], which gives an algorithm to compute the dilation by an hexagonal structuring element for the binary case using a restricted set of directions. On the other hand, this work let us with some open questions. First, what is the "minimal" mutation in the sense of the smallest set of directions one need to follow the evolution of dilation. Second, how can we apply this framework to others morphological operators such as erosion, closings, and openings? Whatever will be the answers, we are deeply conviced of the interest of non-regular analysis in dealing with mathematical morphology.

\section{A Appendix}

Lemma A.1 Consider a compact convex symmetric set $S \subset \mathbb{R}^{n}$ with non empty interior. The map

$$
x \rightsquigarrow \Pi_{K}^{S}(x) \text { is upper semicontinuous. }
$$

For a proof of this lemma, see for instance [7, Aubin-Frankowska].

Lemma A.2 Consider a compact convex symmetric set $S \subset \mathbb{R}^{n}$ with non empty interior such that $j_{S}$ is uniformly differentiable. If $d_{K}^{S}(\cdot)$ is differentiable at a point $x \in K^{c}$, there exists an unique projection $\Pi_{K}^{S}(x)$ and

$$
\nabla d_{K}^{S}(x)=\nabla j_{S}\left(x-\Pi_{K}^{S}(x)\right) .
$$

Proof: Let us consider any $z \in \Pi_{K}^{S}(x)$. Inequality

$$
\frac{d_{K}^{S}(x+h v)-d_{K}^{S}(x)}{h} \leq \frac{j_{S}(x-z+h v)-j_{S}(x-z)}{h}
$$

implies, by taking the limit, that

$$
\limsup _{h \rightarrow 0^{+}} \frac{d_{K}^{S}(x+h v)-d_{K}^{S}(x)}{h} \leq\left\langle\nabla j_{S}(x-z), v\right\rangle
$$

so that

$$
\limsup _{h \rightarrow 0^{+}} \frac{d_{K}^{S}(x+h v)-d_{K}^{S}(x)}{h} \leq \inf _{z \in \Pi_{K}^{S}(x)}\left\langle\nabla j_{S}(x-z), v\right\rangle
$$


Assume now that the norm is uniformly differentiable. First, since $\nabla j_{S}$ is continuous and $\Pi_{K}^{S}$ is upper semicontinuous, we deduce from the Maximum Theorem ${ }^{6}$ that

$$
h \rightarrow \inf _{z_{h} \in \Pi_{K}(x+h v)}\left\langle\nabla j_{S}\left(x-z_{h}\right), v\right\rangle
$$

is lower semicontinuous. Furthermore, since the norm is uniformly differentiable, we know that for any $z_{h} \in \Pi_{K}^{S}(x+h v)$,

$$
\left\{\begin{array}{l}
\frac{d_{K}^{S}(x+h v)-d_{K}^{S}(x)}{h} \geq \frac{j_{S}\left(x-z_{h}+h v\right)-j_{S}\left(x-z_{h}\right)}{h} \\
\geq\left\langle\nabla j_{S}\left(z-z_{h}\right), v\right\rangle-\varepsilon(h) \geq \inf _{z_{h} \in \Pi_{K}^{S}(x+h v)}\left\langle\nabla j_{S}\left(x-z_{h}\right), v\right\rangle-\varepsilon(h)
\end{array}\right.
$$

Therefore, taking the inferior limit in both sides, we obtain the opposite inequality

$$
\liminf _{h \rightarrow 0^{+}} \frac{d_{K}^{S}(x+h v)-d_{K}^{S}(x)}{h} \geq \inf _{z \in \Pi_{K}^{S}(x)}\left\langle\nabla j_{S}(x-z), v\right\rangle
$$

which concludes the proof.

Proof of proposition 3.5: Consider a sequence $x_{n}$ converging to $x \in K^{c}$ such that $d_{K}^{S}(\cdot)$ is differentiable at $x_{n}$. We use lemma A.2 to claim that $\Pi_{K}^{S}\left(x_{n}\right)$ is a singleton and

$$
\nabla d_{K}^{S}\left(x_{n}\right)=\nabla j_{S}\left(x_{n}-\Pi_{K}^{S}\left(x_{n}\right)\right) .
$$

Since $\nabla j_{S}$ is continuous and $\Pi_{K}^{S}$ is upper-semicontinuous (from lemma A.1), we can write

$$
\limsup _{x_{n} \rightarrow x} \nabla j_{S}\left(x_{n}-\Pi_{K}^{S}\left(x_{n}\right)\right)=\nabla j_{S}\left(x-\Pi_{K}^{S}(x)\right),
$$

which together with proposition 3.2 concludes the proof.

Proof of corollary 3.6: We use the fact that, for $x \in K^{c}$,

$$
\nabla j_{B}(x)=\nabla\|x\|=\frac{x-\Pi_{K}^{B}(x)}{d_{K}^{B}(x)}
$$

\footnotetext{
${ }^{6}$ Maximum Theorem Let metric spaces $X, Y$, a set-valued map $F: X \rightsquigarrow Y$ and a function $U: \operatorname{Graph}(F) \mapsto \mathbb{R}$ be given. We associate with them the marginal function $V: X \mapsto \mathbb{R}$ defined by

$$
V(x):=\sup _{y \in F(x)} U(x, y)
$$

- If $U$ and $F$ are lower semicontinuous, so is the marginal function $V$.

- If $U$ and $F$ are upper semicontinuous and if the values of $F$ are compact, so is the marginal function $V$.

The proof is an exercise of topology which is found in many books (for instance [7]).
} 
thanks to proposition 3.5.

Proof of proposition 3.8: Take $p \in \partial d_{K}^{S}(x)$. Obviously, for any $v \in$ $C_{K}^{S}(x)$, we can write

$$
<p, v>\leq C_{\uparrow} d_{K}^{S}(x)(v) \leq 0
$$

Therefore $p \in N_{K}^{S}(x)$.

On the other hand, we have

$$
C_{\uparrow} d_{K}^{S}(x)(v) \leq \limsup _{h \rightarrow 0^{+}, x^{\prime} \rightarrow x} \frac{d_{K}^{S}\left(x^{\prime}+h v\right)-d_{K}^{S}\left(x^{\prime}\right)}{h} \leq \partial j_{S}(v) .
$$

Consequently, for any $v$, we obtain

$$
<p, v>\leq j_{S}(v) .
$$

Hence, since for any $v \in S$ we have $j_{S}(x) \leq 1$, this yields

$$
\sup _{v \in S}<p, v>\leq 1 \text {. }
$$

Therefore $p \in S^{\star}$. This completes the proof.

Proof of corollary 3.8: Let us indicate that, if $S$ is equal to the Euclidean unit ball $B$, we obtain that $S=S^{\star}$, since $\sup _{x \in B}<p, x>=\|p\|$. The proof ensues.

Proof of proposition 5.2: Take $y \in \vartheta_{S}(h, K)$. It is clear that there exists $x \in K$ such that

$$
y=x+\int_{0}^{h} x^{\prime}(s) d s \in x+h S
$$

Consequently $y \in K_{S}(h)$.

Conversely, take $y \in K_{S}(h)$ and consider

$$
y(t)=z+t \frac{y-z}{h}
$$

where $z \in \Pi_{K}^{S}(x)$.

Obviously, we have

$$
y(h)=y, y^{\prime} \in S \text { and } y(0)=z \in K .
$$

Therefore $y \in \vartheta_{S}(h, K)$ and thus

$$
\vartheta_{S}(h, K)=K_{S}(h)
$$

Proof of theorem 5.4: It follows from the fact that

$$
\partial d_{K}^{B}(\cdot) \subset N_{K}(\cdot) \cap B \subset B
$$


and from the proposition 5.3.

Proof of proposition 5.3: Let us take $h>0$ and denote $F(x)=\partial d_{K}^{B}(x)$. First, we will prove that

$$
K(h) \subset \vartheta_{F}(h, K) .
$$

Indeed, consider $y \in K(h)$.

If $y \in K$, it is clear that $y(t)=y$ satisfies

$$
y^{\prime}(t)=0 \in \partial d_{K}^{B}(y(t)) .
$$

Therefore, $y=\vartheta_{F}(h, y) \in \vartheta_{F}(h, K)$.

If $y \in K^{c}$, we consider for $h \geq t \geq 0$,

$$
y(t)=y+t \frac{z-y}{d_{K}^{B}(y)},
$$

where $z \in \Pi_{K}(y)$. Since we have

$$
\frac{z-y(t)}{d_{K}^{B}(y(t))}=\frac{(z-y)\left(\frac{d_{K}^{B}(y)-t}{d_{K}^{B}(y)}\right)}{d_{K}^{B}(y(t))},
$$

and

$$
y(t) \in[y, z] \Longrightarrow \Pi_{K}(y(t))=z \Longrightarrow d_{K}^{B}(y(t))=d_{K}^{B}(y)-t,
$$

we obtain that

$$
\frac{z-y(t)}{d_{K}^{B}(y(t))}=\frac{z-y}{d_{K}^{B}(y)} .
$$

Consequently, by proposition (3.2),

$$
y^{\prime}(t)=\frac{z-y(t)}{d_{K}^{B}(y(t))} \in-F(y(t)) .
$$

Furthermore $y(h)=z \in K$ yields

$$
y \in \vartheta_{F}(h, z) \subset \vartheta_{F}(h, K) .
$$

Hence

$$
K(h) \subset \vartheta_{F}(h, K) .
$$

Moreover, corollary (3.9) implies that

$$
K(h) \subset \vartheta_{F}(h, K) \subset \vartheta_{B}(h, K) .
$$

But, since we also have, by proposition 5.2 ,

$$
\vartheta_{B}(h, K)=K(h)=K \oplus h S,
$$


we obtain, for any $h>0$,

$$
K(h)=\vartheta_{\partial d_{K}^{B}(\cdot)}(h, K) .
$$

Consequently, we obtain

$$
\lim _{h \rightarrow 0^{+}} \frac{d l\left(\vartheta_{\partial d_{K}^{B}(\cdot)}(h, K(0)), K(h)\right)}{h}=0,
$$

which yields

$$
\stackrel{\circ}{K}(0) \ni \partial d_{K(0)}^{B} \cdot
$$

Moreover, since $B$ is convex, we have

$$
K(t+h)=K(t) \oplus h B,
$$

and by the semi-group property for the reachable map, we can generalize the result for any $t>0$, applying the previous ideas iteratively.

\section{References}

[1] L. Alvarez, F. Guichard, P.L. Lions, and J.M. Morel. Axiomatisation et nouveaux opérateurs de la morphologie mathématique. C.R. Acad. Sci. Paris, pages 265-268, t.315, Série I, 1992.

[2] J.-P. Aubin. Viability theory. Birkhauser, Systems and Control : Foundations and Applications, 1991.

[3] J.-P. Aubin. Morphological and Mutational Analysis, Tools for Shape Regulation and Optimization. Commett Matari Programme, CEREMADE, University of Paris-Dauphine, France, 1993.

[4] J.-P. Aubin. Mutational Equations in Metric Spaces. Set-Valued Analysis, 1:pages 3-46, 1993.

[5] J.-P. Aubin. Initiation à l'analyse contemporaine. Masson, Paris, France, 1994.

[6] J.-P. Aubin and A. Cellina. Differential Inclusions (Set-valued maps and viability theory). Springer-Verlag, 1984.

[7] J.-P. Aubin and H. Frankowska. Set-Valued Analysis. Birkhauser, 1990. Systems and Control: Foundations and Applications.

[8] R. van den Boomgaard. Mathematical Morphology: Extensions towards Computer Vision. PhD thesis, Amsterdam University, The Netherlands, Mars 1992. 
[9] R. Brockett and P. Maragos. Evolution equations for continuous-scale morphology. In IEEE Conference on Acoustics, Speech and Signal Processing, San Francisco CA, March 921992.

[10] F.H. Clarke. Optimization and Nonsmooth Analysis. Wiley-Intersience, 1983.

[11] L. Doyen. Évolution, contrôle et optimisation de formes. Thèse de Doctorat, Université Paris-Dauphine, Paris, France, juin 1993.

[12] L. Doyen. Filippov and invariance theorems for mutational inclusions of tubes. Journal of Set-Valued Analysis, 1:283-303, 1993.

[13] L. Doyen. Inverse Function Theorems and Shape Optimization. S.I.A.M Journal on Control and Optimization, (to appear).

[14] L. Doyen. Mutational Equation for Tube and Vison Based Control. Set Valued Analysis, (to appear).

[15] L. Doyen. Shape Lyapunov Functions and visual Servoing. Journal of Mathematical Analysis and Applications, (to appear).

[16] L. Doyen, L. Najman, and J. Mattioli. Mutational Equations of Morphological Dilation Tubes. In J. Serra, editor, Mathematical Morphology and its Application to Signal Processing II, ISMM'94, Fontainebleau, France, september 1994. École des Mines de Paris. (to appear).

[17] H. Frankowska. Control of nonlinear systems and differetial inclusions. Birkhäuser, to appear.

[18] G. Matheron. Random Sets and Integral Geometry. John Wiley and Sons, New York, 1975.

[19] J. Mattioli. Differential Inclusions for Mathematical Morphology. In SPIE: Image Algebra and Morphological Image Processing IV, volume 2030, pages 12-23, San Diego, July 11-16 1993.

[20] J. Mattioli. Differential Relations of Morphological Operators. In Mathematical Morphology and its applications to Signal Processing, pages 162-167, Barcelona, Spain, May, 12-14 1993.

[21] J. Mattioli. Problèmes inverses et relations différentielles en morphologie mathématique. Thèse de Doctorat, Université Paris Dauphine, Paris, France, mai 1993.

[22] J. Mattioli. Relations différentielles d'opérations de la morphologie mathématique. C.R. Acad. Sci. Paris, t. 316, Série I:879-884, 1993.

[23] H. Minkowski. Volumen und Oberfläche. Math. Ann., 57:447-495, 1903. 
[24] L. Najman. Morphologie Mathématique : de la Segmentation d'Images à l'Analyse Multivoque. Thèse de doctorat, Université Paris-Dauphine, Paris, France, Avril 1994.

[25] R.T. Rockafellar and R. Wets. Variational Analysis. Springer-Verlag, 1994. To appear.

[26] M. Schmitt. Des algorithmes morphologiques à l'intelligence artificielle. Thèse Ecole des Mines de Paris, February 1989.

[27] M. Schmitt. Geodesic arcs in non-euclidean metrics: Application to the propagation function. Revue d'Intelligence Artificielle, 3 (2):43-76, 1989.

[28] M. Schmitt and J. Mattioli. Morphologie Mathématique. Logique Mathématiques - Informatique. Masson, Décembre 1993.

[29] J. Serra. Image Analysis and Mathematical Morphology. Academic Press, London, 1982. 